\title{
11 Schlussfolgerungen: Beiträge zum Language Making des Spanischen aus der Berliner Lerngemeinschaft
}

Gemessen an der Größe der weltweiten Sprachgemeinschaft tragen die Spanischlernenden in Berlin und auch die Beschäftigten im Gastgewerbe, die häufig mit Spanischsprachigen in Berührung kommen, nur einen kleinen Teil zum Language Making des Spanischen bei. ${ }^{56}$ Dennoch lohnt es sich, diesen Beitrag genauer in den Blick zu nehmen. Die Befragungen haben gezeigt, dass die Vorstellungen von der Sprache, ihrer Verwendung und ihrem Stellenwert zum Teil weit auseinandergehen, zugleich an einigen Stellen aber auch auffällige Übereinstimmungen bestehen. Interessant ist dabei, dass sich die Lernenden trotz einiger entscheidender Differenzen vollkommen einig sind, dass die Sprache, die sie lernen, Spanisch ist. Dies gilt auch für die gesamte restliche Sprachgemeinschaft, in der die Ansichten ebenfalls breit gestreut sein können und sich von denjenigen der Lernenden sicherlich unterscheiden. Der Prozess des Language Making bringt also zwangsweise Spannungen und konkurrierende Spracheinstellungen mit sich. Was genau Spanisch ist, lässt sich demnach niemals exakt und unveränderlich festlegen. Es handelt sich beim Spanischen - wie bei anderen Sprachen - in den Augen der Sprachgemeinschaft um eine Sprache, ohne dass widerspruchsfrei zu erkennen ist, was diese Sprache exakt ausmacht.

Wichtig ist vor allem den Studierenden die instrumentelle Ebene der Sprache. In den verschiedensten Situationen möchten sie in der Lage sein, mit Hilfe des Spanischen kommunizieren zu können, dabei ihre Netzwerke zu erweitern und neue Kontakte zu entwickeln - zum Teil im beruflichen Bereich, aber auch im privaten Umfeld. Damit gewinnt für sie das Spanische eine wichtige Komponente als Werkzeug, mit dem der Anschluss an die Sprachgemeinschaft gelingen kann. In diesem Punkt stimmen die Studierenden überein mit Gleichaltrigen in den USA. Auch dort betonten Studierende, die Spanisch lernten, in erster Linie die instrumentellen Vorteile der Sprachkenntnisse: Das Erschließen neuer Kommunikationsgemeinschaften und reibungslosere Verständigung mit bestimmten Gruppen (Pomerantz 2002). Auch für andere Sprecherinnen des Spanischen, die damit etwa in Lateinamerika oder in Spanien aufgewachsen sind

56 Die Beiträge in Chiquito/Quesada Pacheco (Hg., 2014) zeigen ein breites Bild der Spracheinstellungen in zahlreichen spanischsprachigen Ländern, aus denen sich zentrale Aspekte des Language Making in der weltumspannenden Sprachgemeinschaft erkennen lassen. 
und es als alltägliches Kommunikationsmittel nutzen, ist die instrumentelle Ebene ihrer Sprache sicherlich ein wichtiges Merkmal. Es fließt dadurch zwar in das Language Making mit ein, ist also ein Teil der kollektiven Vorstellung dessen, was das Spanische ausmacht. Allerdings ist die instrumentelle Eigenschaft der Sprache diesem Teil der Sprachgemeinschaft wahrscheinlich deutlich weniger präsent bzw. sie wird weniger intensiv reflektiert, als dies bei Sprachlernenden der Fall sein dürfte.

Während viele der Befragten im Spanischen ein wirkungsvolles Kommunikationsinstrument sahen, erwarteten sie von der Sprache für sich selbst wenig Grundlagen für materielle Vorteile. Damit widerstehen die Lernenden zum großen Teil einem weit verbreiteten Diskurs, mit dem Sprachen - auch das Spanische - als wichtige ökonomisch verwertbare Ressourcen vermarktet werden (Bruzos Moro 2016). In der starken Betonung der affektiven Dimension des Sprachenlernens unterscheiden sich die Haltungen der Spanischlernenden in Berlin auch von jenen vieler Europäer*innen allgemein: Im Jahr 2012 gaben in der Eurobarometer-Umfrage die meisten Befragten in der EU an, dass sie Sprachen aus beruflichen Gründen oder zur Vorbereitung eines Auslandsstudiums lernten, teils aber auch zum Gebrauch auf Ferienreisen oder zum besseren Verständnis von Menschen anderer Kulturen. Die instrumentelle Dimension war hier durchaus auch stark vorhanden, ${ }^{57}$ aber klar hedonistische Lerngründe wie die Aussagen „for personal satisfaction“ (29\% Zustimmung) oder „to feel more European“ (10\% Zustimmung) lagen im Mittelfeld bzw. am unteren Rand der Skala (EU-Kommission 2012: 62).

Durch seinen vergleichsweise geringen unmittelbaren materiellen Wert (in den Augen der Befragten) erhält das Spanische in seinem Charakter als globale Sprache eine deutlich andere Konnotation als das Englische. Obwohl beide durch ihre Kolonialgeschichte überhaupt erst globalisiert und so auf Basis eines starken Machtgefälles etabliert wurden, können sie ihre Stellung in der Welt heute auf sehr unterschiedliche Weise behaupten. Der Einfluss des Englischen, der häufig weiterhin als kolonial wahrgenommen wird, speist sich zu einem gewichtigen Teil aus seiner ökonomischen Funktion; Skutnabb-Kangas und Phillipson (2010: 79) sprechen hier von einer Entwicklung „from colonization to corporate globalization“. Das Spanische hingegen bezieht seinen Ein-

57 Auch in den Fragestellungen der Eurobarometer-Umfrage gingen materielle und instrumentelle Lernmotivationen fließend ineinander über, etwa beim Item „to get a better job“. Dies kann selbstverständlich klar materiell im Sinne einer besser bezahlten Arbeit gemeint sein, aber auch die generelle Zufriedenheit mit der Arbeit kann eine Rolle spielen. 
fluss in der Welt - und zwar vornehmlich außerhalb seines Kerngebiets - viel deutlicher von nicht-materiellen Faktoren. Dies steht selbstverständlich der Tatsache nicht entgegen, dass in den mehrsprachigen Gesellschaften Lateinamerikas und auch Spaniens das Spanische eine klare sozioökonomische Dominanzstellung mit starker gatekeeping-Funktion gegenüber Minderheiten und indigenen Sprachgemeinschaften innehat (Ricento 2010: 130-133). Auch hier zeigen sich also abweichende oder sogar widersprüchliche Elemente, die zum Language Making des Spanischen beitragen: Einerseits die Konzeptionalisierung als eine Sprache mit geringer ökonomischer Tragkraft, andererseits als Sprache mit klarer sozioökonomischer Vorherrschaft in bestimmten Ländern oder Gesellschaften. Für die weitere Forschung zu Spracheinstellungen gegenüber dem Spanischen zeigt dies das Potenzial von Ansätzen, die Widersprüchlichkeiten herausarbeiten. Kroskrity (2004: 504) spricht von einem „focus on contestation, clashes or disjunctures in which divergent ideological perspectives on language and discourse are juxtaposed.“

Dass die Stärken des Spanische bei den Befragten vor allem auf instrumenteller und affektiver Ebene liegen und weniger im unmittelbar materiellen Bereich, lässt auch sichtbar werden, dass damit vor allem im übertragenen Sinne Kapital angelegt wird. ${ }^{58}$ Bourdieu (2001 [1982]: 78) sieht bei der Beteiligung am linguistischen Markt bei den Sprecher^innen bestimmte „chances de profit matériel et symbolique“, die bei der Nutzung bestimmter sprachlicher Formen zu erwarten sind. Der Wert des Spanischen als Lieferant von symbolischem Profit, etwa kulturellem und sozialem, übersteigt deutlich seine unmittelbar materielle Rendite. Lediglich ausgewählte Privilegierte können unter Umständen von Spanischkenntnissen materiell profitieren. Das Gastgewerbe in Berlin ist ein deutliches Beispiel dafür, wie der ökonomische Nutzen einer sprachlichen Ressource praktisch vollständig extern bleibt, also nicht bei den Sprecher^innen selbst verbleibt. Während sich mehrsprachiger Service durch Kundenzufriedenheit und -bindung für den Betrieb durchaus in Form von gesteigertem Umsatz niederschlagen kann, bleibt von diesem Mehrwert bei den Beschäftigten selbst, also bei den Sprecher^innen, nur äußerst selten ein direkter materieller Nutzen erhalten. Vor diesem Hintergrund muss auch die Aussage von Kroskrity (2004: 501) nuanciert werden, dass Sprachideologien seien „often demonstrably tied to political-economic interests“. Zumindest ist die Betonung auf oft zu legen, denn im vorliegenden Fall stehen direkte politisch-ökonomische Mo-

58 Vgl. auch Vigouroux/Mufwene (2020: 12-13) mit einer kritischen Betrachtung zum Konzept von Sprachen als Kapital. 
tive bei vielen Lernenden nicht im Vordergrund. Dies bedeutet selbstverständlich nicht, dass die Spracheinstellungen und -ideologien der Befragten nicht interessengeleitet oder losgelöst von Machtverhältnissen sind. Die Interessen spielen sich lediglich auf anderen Ebenen ab, etwa beim Wunsch der Teilhabe an einem einfluss- und durchaus prestigereichen Kulturmodell von Hispanidad oder Latinidad.

Sehr positiv waren quer durch alle befragten Gruppen die affektiven und ästhetischen Einschätzungen des Spanischen. Hier schlossen auch die Lernenden in den Volkshochschulen zu den Studierenden auf. An dieser Stelle dürfte ein groBer Teil der Sprachgemeinschaft - inklusive derjenigen im spanischen Sprachgebiet Europas und Lateinamerikas - ähnliche Vorstellungen mit dem Spanischen verbinden. Aus bestehender Forschung lässt sich erkennen, dass romanische Sprachen (neben Spanisch und Französisch beispielsweise auch Italienisch) auch von Muttersprachler^innen in affektiv-ästhetischen Kategorien häufig positiv bewertet werden (Schoel et al. 2013: 34). In diesem Feld von Spracheinstellungen bewegen sich die Lernenden und die bereits bestehende Sprachgemeinschaft also in einem gemeinsamen Diskursrahmen, in dem das Spanische als ansprechende Sprache konstruiert wird. Zum Language Making des Spanischen tragen offensichtlich Lernende wie der Rest der Sprachgemeinschaft den Gedanken bei, dass die Sprache schön ist und das Sprechen oder Erlernen ein positives Erlebnis bietet.

Die Ergebnisse der Befragungen zeigen, wie die Lernenden in Berlin am Language Making des Spanischen teilhaben. Sie schließen sich einer Diskursgemeinschaft an, die sie mitbestimmen und sie formen damit auch das Bild dessen, was in der Welt als Spanisch begriffen wird. Dabei partizipieren sie an größeren sprachideologischen Bezugsrahmen und metasprachlichen Diskursen, die nicht alleine für das Spanische spezifisch sind. Hier spielt beispielsweise die Wahrnehmung unterschiedlicher Schwierigkeit beim Erlernen der beiden Sprachen eine Rolle. Diese kann auf die tatsächliche Lernerfahrung zurückzuführen sein, aber auch auf weit verbreitete Diskurse über den Schwierigkeitsgrad von Sprachen (vgl. erneut Venus 2017, Schwender 2018 für das Französische).

$\mathrm{Zu}$ den größeren Diskursrahmen gehört auch die Muttersprachenideologie, von der zumindest ein Einstellungselement für die Sprachenlehre von einer großen Mehrheit bestätigt wurde. Ideologische Vorstellungen von ,Authentizität‘ in der Sprache werden üblicherweise vor allem mit nicht-standardisierten Formen verbunden, oft etwa bei älteren, weniger mobilen Sprecherinnen aus dem ländlichen Raum (Woolard 2007: 131-132). Beim Fremdspracherwerb verkörpert dagegen eine Lehrperson mit muttersprachlichen Kenntnissen die gewünschte Authentizität, 
auch bei der Vermittlung des Standards. ${ }^{59}$ Sie ist es auch, welche die Sprache aus der Anonymität herausholt, die in der Regel die ideologischen Vorstellungen der großen Standardsprachen prägen: Die erlernte Sprache wird mit einer vermittelnden Person stark verknüpft, von ihr repräsentiert. Sie ist eine Vertreterin dessen, was als genuine (Standard-)Sprachform beispielsweise des Spanischen aufgefasst wird und in diesem Fall durch Authentizität mit Autorität ausgestattet (Paffey 2012: 53). Dies bedeutet aber nicht automatisch, dass die Lernenden auch die Sprachform der Lehrkraft übernehmen. Zwar erwartet man entsprechend der weit verbreiteten Standardsprachideologie von idealen Standardsprecher`innen eine „voz de ningún lugar“, also eine Sprechweise, die von einer klaren geographischen Zuordnung frei bleibt (Woolard 2007: 133). ${ }^{60}$ Dennoch zeigen die Befragungsdaten, dass viele Lernende zumindest eine Präferenz für Sprachformen einer bestimmten Region des Spanischen haben, sei es Lateinamerika oder Spanien. Diese Sprachform kann der Lehrkraft nachempfunden werden, oder aber aus anderen Quellen wie Lehrwerken und Medien oder von eigenen spanischsprachigen Kontaktpersonen im Bekannten- und Freundeskreis. Wenn „die Plurizentrik des Spanischen eine diskursiv konstruierte Vielheit ist und kein Nebeneinander, das sich auf geographisch und politisch abgrenzbare Nationalstaaten bezieht“ (Kailuweit 2015: 112), dann können an dieser diskursiven Konstruktion selbstverständlich auch die Lernenden in Berlin teilhaben und mitwirken. In welchem Maße die Lernenden das Spanische tatsächlich im Erwerbsprozess gezielt lokalisieren möchten, ob und wie sie sich im plurizentrischen Variationsfeld positionieren, diesen Fragen sollten weitere Forschungsarbeiten nachgeheneine (vgl. die Beiträge in Leitzke-Ungerer/ Polzin-Haumann Hg., 2017).

Die zunehmende Präsenz des globalen Spanischen folgt in Berlin vor allem dem grassroots-Mechanismus nach Mar-Molinero (2008, 2010: 169): Es herrscht eine starke Nachfrage vor, die im klassischen Bildungssektor noch nicht vollständig befriedigt wird, so dass institutionelle Akteure bisher das Spanische weniger deutlich befördern als von der Lerngemeinschaft gewünscht oder eingefordert. Französisch ist im Schulwesen weiterhin sehr präsent; das Spanische tritt hinzu, bleibt aber insbesondere in der Erwachsenenbildung stark (vgl. auch Grünewald/Küster 2017:

59 Zum Konzept der Authentizität im Fremdsprachunterricht vgl. umfassender auch die Beiträge in Frings / Leitzke-Ungerer (Hg., 2010). Melo-Pfeifer (2019: 23-24) zeigt die hohen Selbstansprüche und auch Unsicherheiten angehender Lehrkräfte für Spanisch und Französisch an ihre eigenen Sprachkenntnisse, etwa gegenüber Muttersprachler*innen.

60 Dass der Standard dennoch in vielen Fällen klar geographisch markiert ist (die Pariser Norm des Französischen ist ein einschlägiges Beispiel), dass das Sprechen des Standards zudem jederzeit auch sozial markiert ist und historisch gebunden, wird in der ideologischen Repräsentation oft ausgeblendet. 
15-16). Die Zukunftsperspektiven des Spanischen als Fremdsprache im deutschsprachigen Raum stellen Klump und Willems (2012: 169) folgendermaßen dar:

Wenngleich sich dies [die Erfolgsgeschichte, P.K.] nicht mit Bestimmtheit voraussagen lässt, so erscheinen doch die gegenwärtigen Rahmenbedingungen hierfür mehr als günstig. Schließlich handelt es sich um eine von zahlreichen Institutionen geförderte Weltsprache mit positivem Image, deren Erlernen vielseitig motiviert und im Bildungssystem mittlerweile fest verankert ist.

Trotz dieses grassroots-Charakters des Spanischlernens scheinen die Lernenden ihre eigene Rolle im Language Making noch als eher gering einzuschätzen bzw. sich ihres mitformenden Anteils nicht unbedingt bewusst zu sein. Auch wenn das globale Spanische sicher als eine „voz pública y anónima“ (Woolard 2007: 135) gelten kann, empfinden sich die Lernenden selbst nicht ohne Weiteres als einflussreicher Teil dieser anonymen Öffentlichkeit. Wie beispielsweise die gefestigte Muttersprachenideologie zeigt, sehen sie sich eher nicht als Akteure, die mit darüber bestimmen dürfen, was das Spanische ist und wie es zukünftig aussehen wird (vgl. die Frage des ownership von Sprachen, Heller 2010: 106-107). Dass sie dies selbstverständlich dennoch tun, ergibt sich aber aus der ständigen Interaktion mit der Sprachgemeinschaft. Viele der Befragten waren bereits in spanischsprachigen Ländern, haben spanischsprachige Kontakte und nutzen ihre Sprachkenntnisse. Damit nehmen sie ganz automatisch teil an der Weiterentwicklung der Diskursformation, die wir Spanisch nennen. Die Entwicklung hin zu einem wahrnehmbaren Diskurs des Spanischen als „lengua del mestizaje“, der sich von Erzählungen der Reinheit und Ursprünglichkeit zunehmend entfernt, kann die offen anerkannte Teilhabe der Lernenden am Language Making künftig erleichtern (Fernández 2007). Mag auch selbst für das Englisch dieser Prozess noch lange nicht abgeschlossen sein (und noch weniger für das Französische), so scheint das Global Spanish in dieser Hinsicht einem ähnlichen Pfad zu folgen. 